\title{
Bronchial artery embolisation for the treatment of massive haemoptysis resulting from pulmonary tuberculosis
}

\author{
I Carim, MB BCh, FCRad(D) \\ H Carim, MB BCh, DCH (SA) \\ M Modi, MB BCh, MMed, FCRad(D)
}

Division of Radiology, Department of Radiation Sciences, Chris Hani Baragwanath Hospital and the University of the Witwatersrand, Johannesburg

\begin{abstract}
Objectives. To report our experience with bronchial artery embolisation (BAE) for the treatment of massive haemoptysis due to untreated or partially treated pulmonary tuberculosis.

Materials and methods. In February 2007 five male patients, between the ages of 40 and 51, presented to Chris Hani Baragwanath Hospital (CHBH) with massive haemoptysis. Bronchial arteriography and embolisation were performed using a 4 French $\mathrm{C} 2$ catheter and polyvinyl alcohol (PVA) particles ranging from 300 to 900 micrometers.

Results. Seven bronchial arteries in total were embolised (2 patients required embolisation of 2 arteries each). The haemoptysis was controlled during the first endovascular treatment session in all 5 patients. No serious complications developed in any of our patients as a result of the interventions. At the 1-month follow-up none of the patients had reported any episodes of haemoptysis.

Conclusion. Massive haemoptysis due to pulmonary tuberculosis can be effectively treated with BAE when in a controlled environment, with the proper patient selection and with the appropriate expertise.
\end{abstract}

\section{Introduction}

Percutaneous transcatheter embolisation is a safe and effective method of vascular occlusion that has been successfully applied in practically every vascular territory to arrest haemorrhage. It has become the therapeutic technique of choice in the treatment of many vascular abnormalities. ${ }^{1}$

Bronchial artery embolisation (BAE) is a safe and well-accepted procedure for the management of massive or recurrent haemoptysis. ${ }^{2}$
Massive haemoptysis is defined as the expectoration of more than 300 $\mathrm{ml}$ of blood in a 24-hour period. ${ }^{2}$ The mortality rate from untreated massive haemoptysis can be as high as $50 \% .^{3}$ Death, in most cases, is secondary to asphyxiation from aspiration rather than from the bleeding itself. Surgical management during an acute episode of massive haemoptysis also has a high mortality rate, reported to be in the region of $30-40 \%{ }^{4}$

We report our experience with BAE for the immediate treatment of massive haemoptysis due to untreated or partially treated pulmonary tuberculosis (PTB) at Chris Hani Baragwanath Hospital (CHBH). $\mathrm{CHBH}$ is a 3200 -bed public-sector urban hospital in Johannesburg, South Africa. It is affiliated to the University of the Witwatersrand and services the 3 million-plus inhabitants of Soweto.

\section{Patients and methods}

In February 2007, five male patients between the ages of 40 and 51, presented to the respiratory unit with massive haemoptysis. They were then referred to our unit for BAE. In all these patients the cause of the haemoptysis was determined to be untreated or partially treated PTB (Table I). Where available, the HIV status of the patients was also documented.

For logistical reasons bronchoscopy was not performed before embolisation, therefore the decision to perform angiographic intervention was made on the basis of review of chest radiographs alone. These were evaluated for the site and extent of the pathology. In all the patients referred to us, chest radiographs demonstrated features of advanced, extensive and chronic disease, seen commonly with PTB (Figs la and b).

Four of the 5 patients were stable after having received blood transfusions. The fifth patient was sent to our unit intubated and on ventilatory support.

In all 5 patients, the right femoral artery was punctured using the Seldinger technique. Initially non-selective aortograms were performed using a 5 French pigtail catheter, followed by selective and super-selective bronchial artery catherisation using a 4 French Cobra 2 catheter to accurately detect the site of haemorrhage.

\begin{tabular}{|c|c|c|c|}
\hline \multicolumn{4}{|c|}{ Table I. PTB and HIV status } \\
\hline & Pulmonary tuberculosis status & Retroviral status & CD4 counts $\left(\right.$ cells $\left./ \mathrm{mm}^{3}\right)$ \\
\hline Patient 1 & Partially treated PTB, bronchiectasis & Positive & 194 \\
\hline Patient 2 & Partially treated PTB, previous defaulter & Unknown & - \\
\hline Patient 3 & Untreated PTB & Positive & 376 \\
\hline Patient 4 & Third episode of partially treated РТВ & Positive & 34 \\
\hline Patient 5 & Untreated PTB & Unknown & - \\
\hline
\end{tabular}




\section{ORIGINAL ARTICLE}

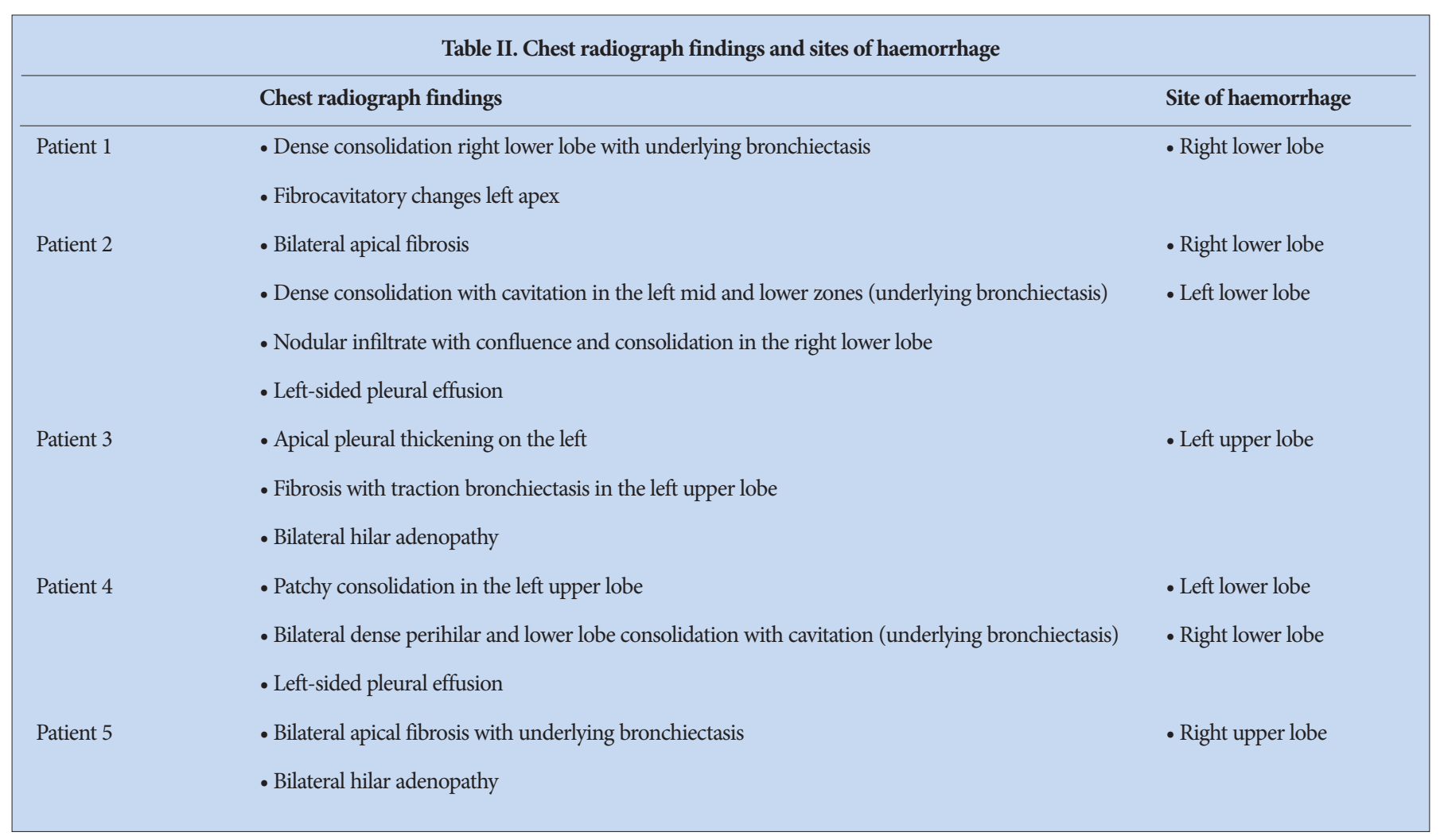

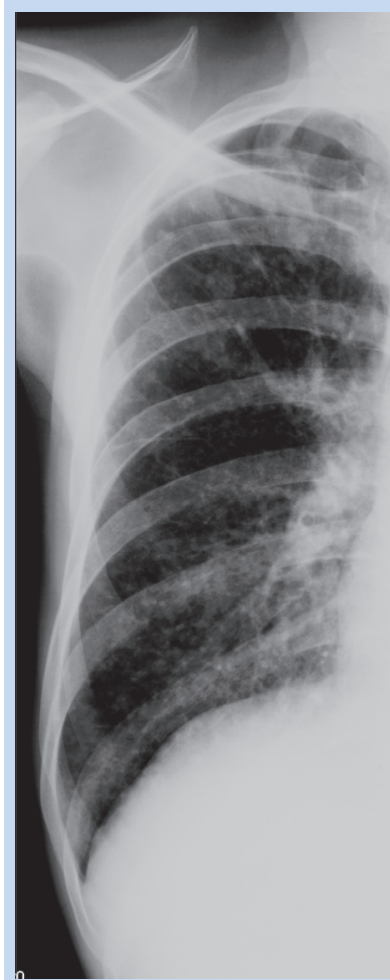

Fig. 1a. Chest radiograph of patient 2 demonstrating: bilateral apical fibrosis with bronchovascular distortion and volume loss, a diffuse nodular infiltrate with confluence and consolidation in the right lower lobe, dense consolidation with underlying bronchiectasis in the left mid and lower zones and a left-sided pleural effusion.

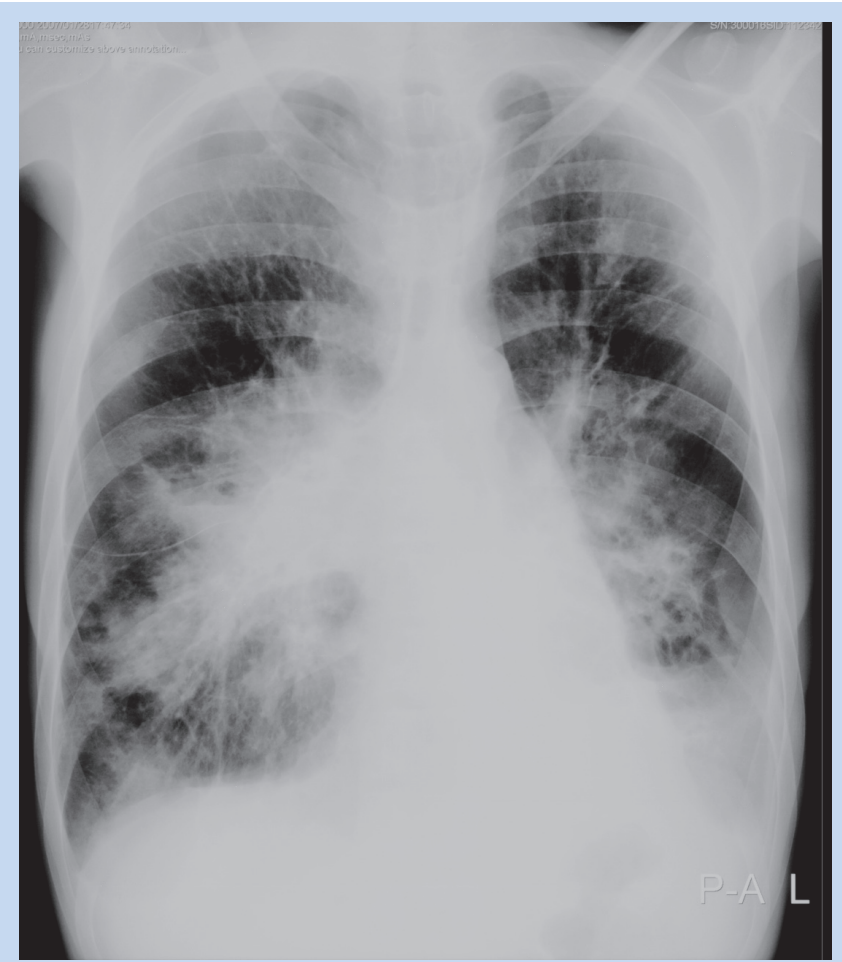

Fig. 1b. Chest radiograph of patient 4 demonstrating: bilateral dense perihilar and lower lobe consolidation with cavitation and underlying bronchiectasis, patchy consolidation in left upper lobe and a left-sided pleural effusion. 


\section{ORIGINAL ARTICLE}

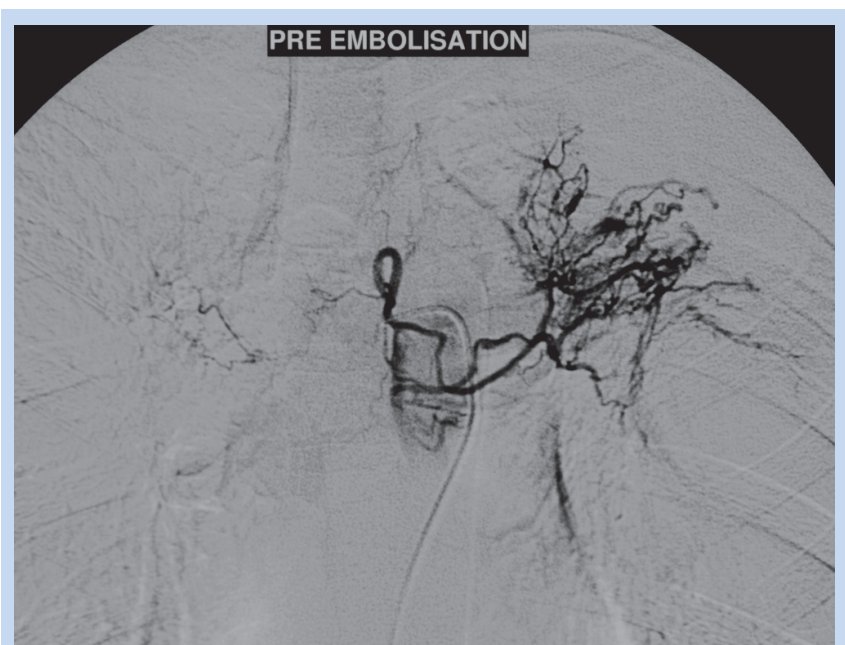

Fig. 2. Bronchial arteriography demonstrating hypertrophy of the bronchial artery with hypervascularity.

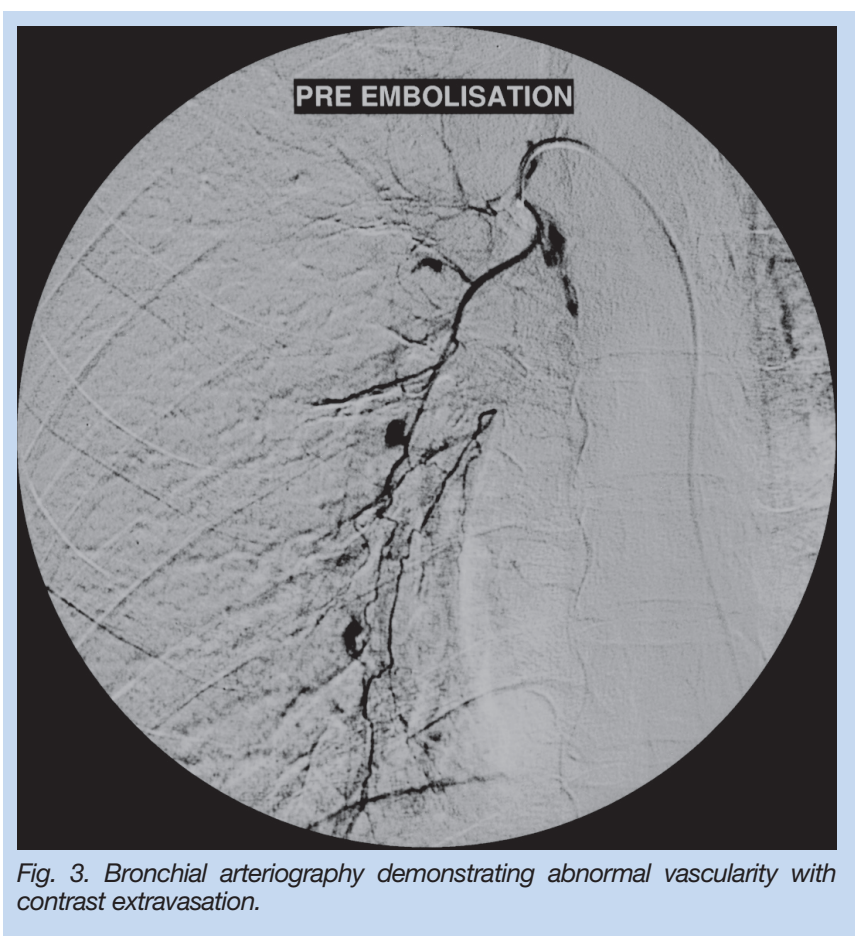

The sites of haemorrhage varied from patient to patient (Table II).

In 4 out of the 5 patients the same $\mathrm{C} 2$ catheter that was used to detect the site of haemorrhage was also used for the embolisation. In 1 case a 4 French Sheppard Hook catheter was used due to difficulty in accessing the bronchial arteries. Micro-catheters, though routinely used in our centre for BAE and definitely recommended and preferred, were unavailable at the time.

Hypervascularity was a common finding at the site of bleeding in all of the 7 arteries embolised ( 2 patients required embolisation of 2 arteries each). Hypertrophy of the bronchial artery was seen in 4 cases and extravasation of contrast into the bronchial lumen was seen in 1 case (Figs 2 and 3 ).

In 1 patient we encountered a spinal artery being fed by a branch

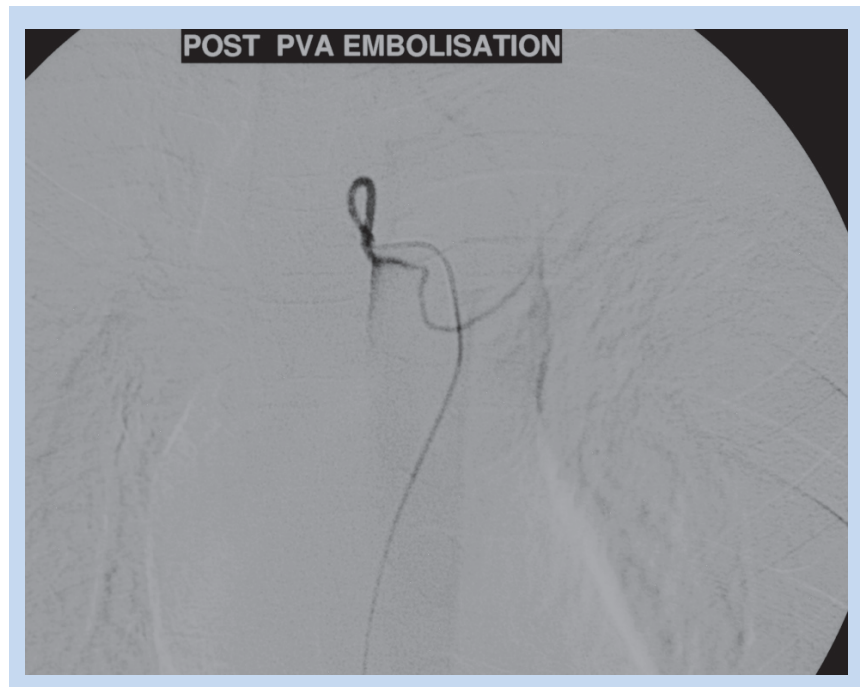

Fig. 4. Post embolisation bronchial arteriography demonstrating occlusion of abnormal vessels.

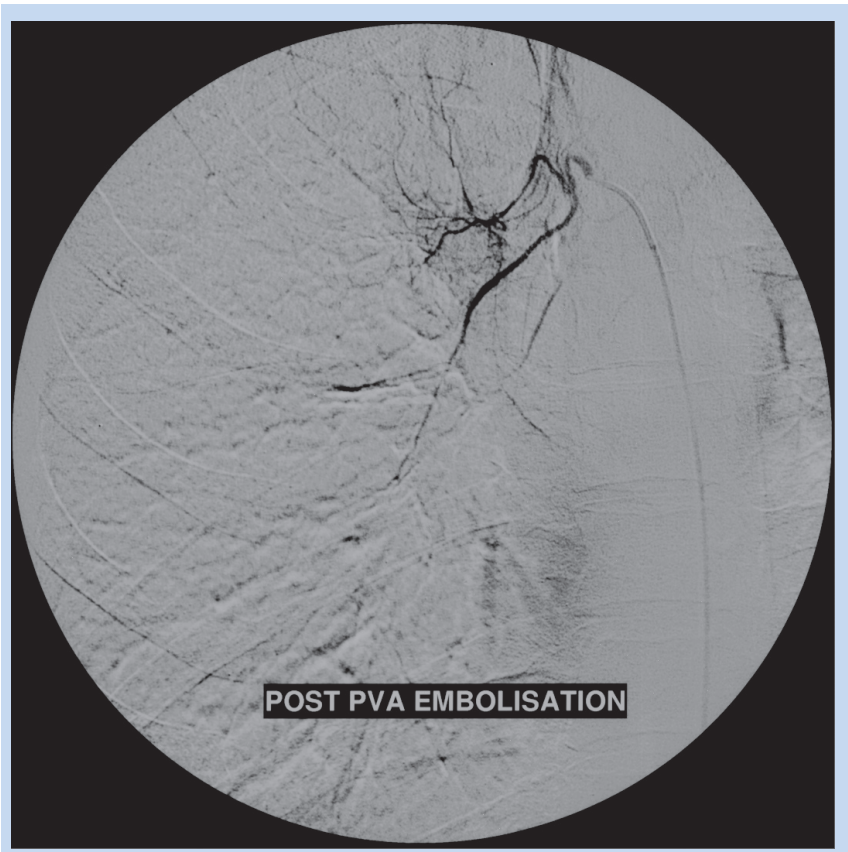

Fig. 5. Cessation of bleeding following embolisation..

of the proximal bronchial artery. Here a selective embolisation was performed with the catheter positioned distal to the origin of the feeding vessel.

Polyvinyl alcohol (PVA) particles ranging from 300 - 500 micrometers up to 700 - 900 micrometers were used in all cases. These were gradually injected into the bronchial arteries with a dilute mixture of contrast using a $10 \mathrm{ml}$ syringe.

\section{Results}

Seven bronchial arteries in total were embolised (2 patients required embolisation of 2 arteries each). Embolisation was considered to be successful if less than $100 \mathrm{ml}$ of blood was expectorated within 24 hours following the procedure. Thereafter, occasional bloodstained sputum, or 
none at all, was considered to indicate a successful outcome.

The haemoptysis was controlled during the first endovascular treatment session in all 5 patients; 2 patients having minimal haemoptysis $(<100 \mathrm{ml}$ in 24 hours) and 3 patients having no haemoptysis at all (Figs 4 and 5). No serious complications developed in any of our patients as a result of the interventions, although 1 patient did complain of chest pain corresponding to the vascular territory embolised. The patient was treated with non-steroidal anti-inflammatory drugs and the pain subsided within 24 hours.

At the 1-month follow-up none of the patients had reported any episodes of haemoptysis.

\section{Discussion}

Bronchial arteriography was first performed in the late 1950s and early 1960s using non-selective aortography, while the first selective bronchial angiogram was performed in 1963 . The Remy group performed the first BAE for haemoptysis in $1973 .{ }^{5}$ Since then many studies have documented the use of BAE for the effective treatment of haemoptysis. ${ }^{6,7}$

The haemoptysis may result from various causes and differs greatly between the Western and non-Western world. In South Africa PTB, particularly tuberculous bronchiectasis is the most common underlying cause of massive haemoptysis. ${ }^{8,9}$ Although PTB is effectively treated with chemotherapy in the majority of patients, $10-20 \%$ will develop serious complications. ${ }^{6}$ This percentage is increased substantially in our population because of the complex socio-economic circumstances together with a lack of insight into the disease that often leads to incomplete or partial treatment in a large number of patients (Fig. 6).

The prevalence of tuberculosis in sub-Saharan Africa has also been on the increase due largely to the advent of the HIV epidemic. ${ }^{10}$ As a result we are now encountering more life-threatening complications of the disease, and post-tuberculous bronchiectasis is the major cause of massive haemoptysis in Southern Africa. ${ }^{9}$

Small, single episodes of haemoptysis and chronic minor haemoptysis associated with PTB, though an important clinical problem, are generally managed well with anti-TB drugs. On the other hand, massive haemoptysis can be life threatening because of either volume depletion and circulatory collapse or, more often, aspiration and consequent asphyxiation. In this setting urgent intervention is required due to the high mortality rate (reportedly between $50 \%$ and $100 \%$ ) associated with conservative management. ${ }^{11}$

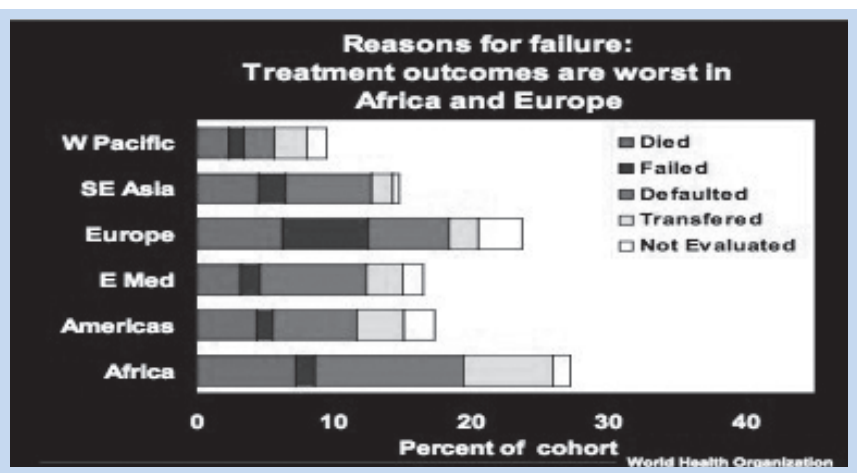

Fig. 6. Treatment outcomes of PTB in Africa compared with the rest of the world World Health Organization, 2001).
Surgery during an acute episode of massive haemoptysis is also associated with a high morbidity and mortality rate. ${ }^{8} \mathrm{BAE}$ is the preferred method of treatment for massive haemoptysis in patients who have diffuse lung disease or those who are not surgical candidates. ${ }^{12} \mathrm{BAE}$ should be considered early in the evolution of massive haemoptysis.

BAE for the short-term control of haemoptysis is effective in around $90 \%$ of cases. Incomplete embolisation is usually the reason for early rebleeding. Ten to twenty per cent of patients will develop late rebleeding within 1 year due to the proliferation of collateral vessels. Repeated embolisation may be attempted for early or late rebleeding, however patients with localised lesions and adequate pulmonary function reserve should undergo surgical resection, as this is the most effective definitive therapy. ${ }^{12}$

In $90 \%$ of cases the source of haemoptysis is the bronchial circulation. ${ }^{13}$ Thus familiarity with the vascular anatomy is important, especially since the anatomy of the bronchial arteries can be variable. The arteries usually arise directly from the proximal descending aorta. A useful landmark for accessing the arteries is in the region of the origin of the left main bronchus, most commonly between the levels of the $\mathrm{T} 5$ and $\mathrm{T} 6$ vertebrae. Based on a study of 150 human cadavers in 1948 Cauldwell et al. ${ }^{14}$ reported 4 classic bronchial artery branching patterns (Fig. 7).

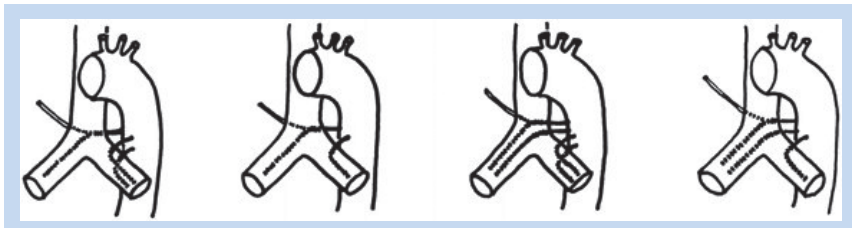

Fig. 7. Diagrams illustrating the different types of bronchial arterial supply: Type I, two bronchial arteries on the left and one on the right that manifests as an ICBT (intercostal bronchial trunk); Type II, one on the left and one ICBT on the right; Type III, two on the left and two on the right (one ICBT and one bronchial artery); Type IV, one on the left and two on the right (one ICBT and one bronchial artery).

It is important to note that in some cases branches of the bronchial artery supply the spinal artery. This makes it imperative to assess the bronchial circulation prior to embolisation in order to ensure that the spinal arteries are not inadvertently embolised. In these instances it is essential to perform the embolisation distal to the origin of the branches to the spinal artery.

\section{Conclusion}

Massive haemoptysis due to PTB can be effectively treated with BAE when in a controlled environment, with the proper patient selection and with the appropriate expertise. It should not be considered definitive treatment in patients with lesions that are appropriate for surgical resection, as there is a significant recurrence rate associated with the procedure.

1. Drooz AT, Lewis CA, Allen TE, et al. Quality improvement guidelines for percutaneous transcatheter embolisation. J Vasc Interv Radiol 2003; 14:S237-S242.

2. Hayakawa K, Tanaka F, Torizuka T, et al. Bronchial artery embolisation for haemoptysis: immediate and long-term results. Cardiovasc Intervent Radiol 1992; 15:154-159.

3. Jean-Baptiste, E. Clinical assessment and management of massive haemoptysis. Crit Care Med 2000; 28:1642-1647.

4. Knott-Craig CJ, Oostuizen JG, Rossouw G, et al. Management and prognosis of massive haemoptysis. Thorac Cardiovasc Surg 1993; 105:394-397.

5. Remy J, Voisin C, Dupuis C, et al. Traitement des hemoptysies par embolization de la circulation system- 
ique. Ann Radiol (Paris) 1974; 17:5-16.

6. Uflacker R, Kaemmerer A, Neves C, Picon PD. Management of massive haemoptysis by bronchial artery embolisation. Radiology 1983; 146:627-637.

7. Remy J, Arnaud A, Fardou H, et al. Treatment of haemoptysis by embolisation of bronchial arteries Radiology 1977; 122:33-37.

8. Conlan AA, Hurwitz SS, Krige L, et al. Massive haemoptysis: review of 123 cases. J Thorac Cardiovasc Surg 1983; 85(1): 120-124

9. Wong ML, Szkup P, Hopley MJ. Percutaneous embolotherapy for life-threatening haemoptysis. Chest 2002; 121:95-102.
10. Aaron L, Saadoun D, Calatroni I, et al. Tuberculosis in HIV-infected patients: a comprehensive review. Clin Microbiol Infect 2004; 10:388-398

11. Najarian KE, Morris CS. Arterial embolization in the chest. I Thorac Imaging 1998; 13:93-104.

12. Johnson LJ. Manifestations of haemoptysis. Postgrad Med 2002; 112: 101-113.

13. Remy J, Remy-Jardin M, Voisin C. Endovascular management of bronchial bleeding. In: Butler J, ed. The Bronchial Circulation. New York: Dekker, 1992: 667-723.

4. Cauldwell E, Siekert R, Lininger R, et al. The bronchial arteries: an anatomic study of 105 human cadavers. Surg Gynecol Obstet 1948; 86:395-412.

\section{Clinical Magnetic Resonance Imaging e-dition, 3rd edition}

Text with Continually Updated Online Reference, 3-Volume Set

By Robert R. Edelman; John R. Hesselink; Michael B. Zlatkin and John V. Crues, III, ISBN 1416030816 / 9781416030812 .

Book/Electronic Media

Saunders · Published November 2005

Health \& Medical Publishing Group

Private Bag X1, Pinelands, 7430

Tel: $021-6578200$ - Fax: 0866950461

e-mail: claudec@hmpg.co.za I

brents@hmpg.co.za
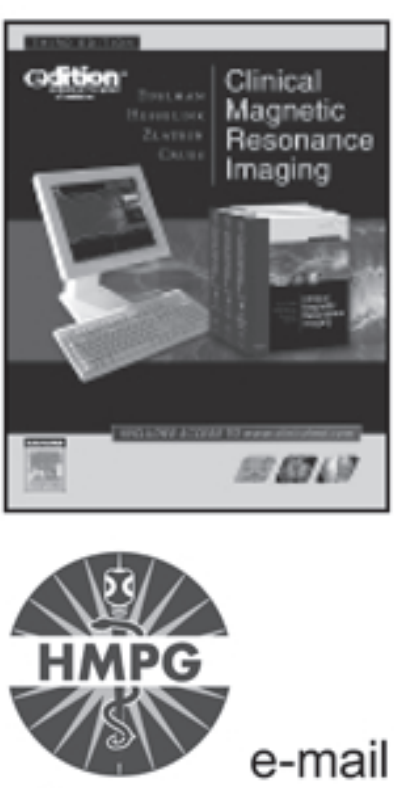

brents@hmpg.co.za for your PIN entitling you to a FREE 28 day trial of the e-dition website!!!

The MRI reference that the American Journal of Roentgenology called 'hard to beat' is back in a state- of-the-art New Edition! It comprehensively examines all of the newest technologies and clinical applications relevant to MR imaging of the heart, brain, head and neck, spine, body, and musculoskeletal system. 4,700 beautifully reproduced illustrations, including hundreds of new full-color images, help readers accurately diagnosis a broad spectrum of conditions. This exhaustively revised 3rd Edition delivers more than $70 \%$ new content and authors as well as a new full-color format that covers all the important technologies as you see them. The associated website is available from any internet access with a username and password. On it, you'll find the entire text online; all 4,700 images and illustrations ready to download into presentations; weekly content updates personally overseen by the editors; current protocols; case studies, including hundreds of images not found in the text; video clips demonstrating key MR approaches and techniques; links from all the references to Medline; and more! 